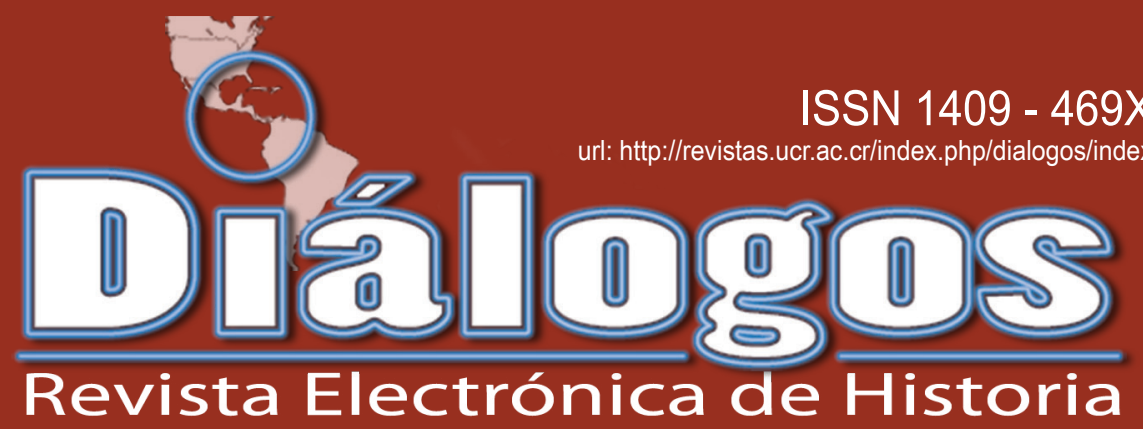

Escuela de Historia. Universidad de Costa Rica Volumen』ם [ especial en homenaje a Víctor Hugo Acuña. Octubre 2013

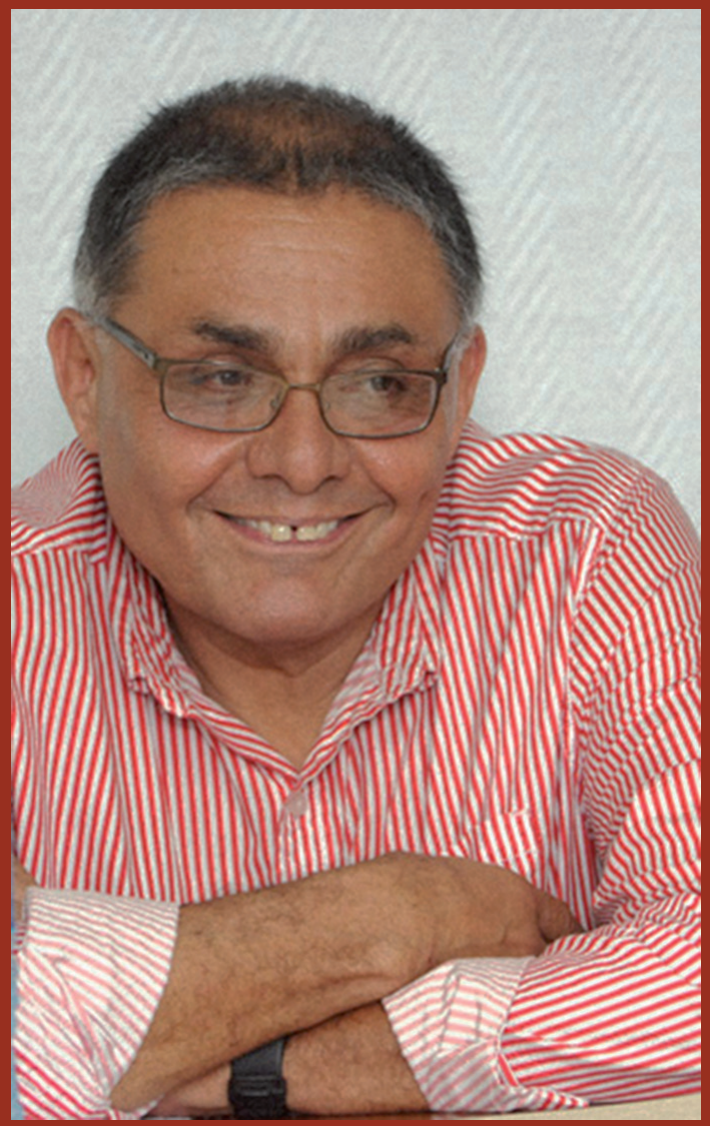

Director de la Revista: Dr. Juan José Marín Hernández juan.marinhernandez@ucr.ac.cr

Editor académico: Dr. Ronny Viales Hurtado - ronny.viales@ucr.ac.cr Editor técnico: M.Sc. Marcela Quirós G. - marcela.quiros@ucr.ac.cr 



\section{Miembros del Consejo Editorial:}

Dr. Juan José Marín Hernández, Catedrático. Director del Centro de Investigaciones Históricas de América Central. Universidad de Costa Rica. Costa Rica. juan. marin@ucr.ac.cr

Dr. Ronny Viales Hurtado. Catedrático. Historia Económica y Social. Universidad de Costa Rica. Director de la Escuela de Historia. Costa Rica. ronny. viales@ucr.ac.cr

Dr. David Díaz Arias: Catedrático. Historia Política, Director del posgrado de Historia y Docente de la Escuela de Historia, Universidad de Costa Rica, Costa Rica.david.diaz@ucr.ac.cr

MSc. Francisco Enríquez. Historia Social. Universidad de Costa Rica. Costa Rica. francisco.enriquez@ucr. ac.cr

Dra. Ana María Botey. Historia de los movimientos sociales. Universidad de Costa Rica. Costa Rica. abotey@gmail.com

\section{Miembros del Consejo Asesor Internacional:}

Dr. José Cal Montoya. Universidad de San Carlos de Guatemala.Guatemala. jecalm@correo.url.edu.gt

Dr. Juan Manuel Palacio. Universidad Nacional de San Martín. Argentina.jpalacio@unsam.edu.ar

Dr. Eduardo Rey. Universidad de Santiago de Compostela. España. ereyt@usc.es

Dr. Heriberto Cairo Carou. Departamento de Ciencia Política y de la Administración III Universidad Complutense de Madrid. España. hcairoca@cps.ucm.es

Dra. Rosa de la Fuente. Departamento de Ciencia Política y de la Administración III Universidad Complutense de Madrid. España. rdelafuente@cps. ucm.es

Dr. Javier Franzé. Departamento de Ciencia Política y de la Administración III Universidad Complutense de Madrid. España. javier.franze@cps.ucm.es

Dr. Jaime Preciado Coronado Departamento de Estudios Ibéricos y Latinoamericanos. Universidad de Guadalajara. México. japreco@hotmail.com
Dr. Gerónimo de Sierra. Vicerrector de la Universidade Federal da Integração Latino-Americana (UNILA) y Departamento de Sociología, Facultad de Ciencias Sociales de la Universidad de la República. Uruguay. geronimo@fcs.edu.uy

Dr. Antonio Palazuelos. Departamento de Ciencia Política y de la Administración III - Universidad Complutense de Madrid. España. palazuelosa@cps. ucm.es

Dr. Werner Mackenbach. Universidad Potsdam. Alemania. werner.mackenbach@uni-potsdam.de

Dr. Guillermo Castro. Ciudad del Saber Panamá. Panamá.gcastro@cdspanama.org

Dra. Natalia Milanesio. University of Houston. Estados Unidos.nmilane2@Central.UH.EDU

Dr. Ricardo González Leandri. Consejo Superior de Investigaciones Científicas - España. España. rgleandri@gmail.com

Dra. Mayra Espina. Centro de Estudios Psicológicos y Sociológicos, La Habana. Cuba.mjdcips@ceniai.inf.cu

Dra. Montserrat Llonch. Departamento de Economía e Historia Económica Universidad Autónoma de Barcelona. España.montserrat.llonch@uab.es

Dra. Estela Grassi. Universidad de Buenos Aires. Argentina. estelagrassi@gmail.com

Dra. Yolanda Blasco. Universidad de Barcelona. España. yolandablasco@ub.edu

Dr. Alfredo Falero. Departamento de Sociología. Universidad de la República. Uruguay. alfredof@adinet. com.uy

\section{Portada:}

Fotografía de Anel Kenjekeeva, Oficina de Divulgación Universidad de Costa Rica, publicada en http://www. ucr.ac.cr/noticias/2012/09/13/escuela-de-historia-rindehomenaje-a-victor-hugo-acuna.html

\section{Equipo Técnico Editorial:}

$\begin{array}{ll}\text { Editora Técnica: } & \text { M.Sc. Marcela Quirós Garita. } \\ & \text { marcela.quiros@ucr.ac.cr } \\ \text { Diagramación: } & \text { Cindy Chaves Uribe } \\ \text { Asistentes: } & \begin{array}{l}\text { Pablo Hurtado Granados } \\ \text { Maureen Méndez Montero }\end{array}\end{array}$


"Diálogos Revista Electrónica de Historia" se publica desde octubre de 1999.

\section{Diálogos está en los siguientes repositorios:}

Dialnet

http://dialnet.unirioja.es/servlet/

revista?tipo_busqueda=CODIGO\&clave_revista $=3325$

Latindex

http://www.latindex.unam.mx/larga.php?opcion=1\&folio=12995;

\section{UCRindex}

http://www.revistas.ucr.ac.cr/

\section{Scielo}

http://www.scielo.cl/

\section{eRevistas}

http://www.erevistas.csic.es/

\section{REDALYC}

http://redalyc.uaemex.mx/src/inicio/FrmBusRevs2.jsp?iEdoRev=2\&cvepai=11;

\section{LANIC}

http://lanic.utexas.edu/la/ca/cr/indexesp.html

Repositorio de Revistas Universidad de Costa Rica http://www.latindex.ucr.ac.cr/

Directorio y recolector de recursos digitales del

Ministerio de Cultura de España

http://roai.mcu.es/es/inicio/inicio.cmd

DOAJ Directory of open access \& Hybrid journals

http://www.doaj.org/doaj?func=byTitle\&hybrid=1\&query=D

Biblioteca de Georgetown

http://library.georgetown.edu/newjour/d/msg02735.html

Asociación para el Fomento de los Estudios Históricos

en Centroamérica

http://afehc. apinc.org/index.php?action=fi_aff\&id=1774

Universidad de Saskatchewan, Canadá

https://library.usask.ca/ejournals/view/1000000000397982

\section{Monografias}

http://www.monografias.com/Links/Historia/more12.shtml

\section{Hispanianova}

http://hispanianova.rediris.es/general/enlaces/hn0708.htm

Universidad del Norte, Colombia

http://www.uninorte.edu.co/publicaciones/memorias/enlaces.htm

Universidad Autónoma de Barcelona

http://seneca.uab.es/historia/hn0708.htm

Repositorio Invenia - Gestión del Conocimiento

http://www.invenia.es/oai:dialnet.unirioja.es:ART0000086144

\section{Enlace Académico}

http://www.enlaceacademico.org/biblioteca/

revistas-en-formato-digital-centroamerica/

\section{Electronic Resources}

http://sunzi1.lib.hku.hk/ER/detail/hkul/3987318

Revistas académicas en texto completo

http://web.prw.net/ vtorres/
Diálogos se anuncia en las siguientes

instituciones y sitios académicos:

Maestroteca

http://www.maestroteca.com/detail/553/dialogos-revista-electronica-de-historia.html

Biblioteca de Georgetown

http://library.georgetown.edu/newjour/d/msg02735.html

Asociación para el Fomento de los Estudios Históricos en Centroamérica

http://afehc. apinc.org/index.php?action=fi_aff\&id=1774

Universidad de Saskatchewan, Canadá

https://library.usask.ca/ejournals/view/1000000000397982

\section{Monografias}

http://www.monografias.com/Links/Historia/more12.shtml

\section{Hispanianova}

http://hispanianova.rediris.es/general/enlaces/hn0708.htm

Universidad del Norte, Colombia

http://www.uninorte.edu.co/publicaciones/memorias/enlaces.htm |

Universidad Autónoma de Barcelona

http://seneca.uab.es/historia/hn0708.htm

Repositorio Invenia - Gestión del Conocimiento

http://www.invenia.es/oai:dialnet.unirioja.es:ART0000086144

\section{Enlace Académico}

http://www.enlaceacademico.org/biblioteca

revistas-en-formato-digital-centroamerica/

\section{Electronic Resources}

http://sunzi1.lib.hku.hk/ER/detail/hkul/3987318

Revistas académicas en texto completo

http://web.prw.net/ vtorres/

La revista electrónica Diálogos es financiada por

Vicerrectoría de Investigación de la Universidad de Costa Rica
Dialnet - eRevistas

- UCRindex - Latindex -

REDALYC - DOJAC - Directorio

y recolector de recursos

digitales del Ministerio de

Cultura de España

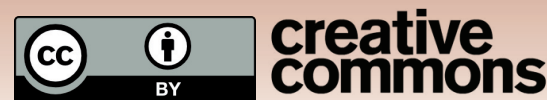



HISTORIA DE

COSTA RICA 



\title{
ANÁLISIS COMPARATIVO DEL IMPACTO Y PERCEPCIONES SOBRE EL TURISMO DE LOS POBLADORES DE LAS COMUNIDADES DE BARRIO EL CARMEN Y JUANITO MORA EN PUNTARENAS, COSTA RICA 2011
}

\section{COMPARATIVE ANALYSIS OF THE IMPACT AND PERCEPTIONS ON TOURISM OF THE RESIDENTS OF THE COMMUNITIES OF BARRIO EL CARMEN AND JUANITO MORA IN PUNTARENAS, COSTA RICA 2011}

Jorge Bartels Villanueva

\begin{abstract}
Palabras claves
Turismo, ingresos, población económicamente activa, Puntarenas, Barrio el Carmen, Juanito Mora, hogares, cultura.
\end{abstract}

\section{Keywords}

Tourism, income, labor force, Puntarenas, Barrio El Carmen, Juanito Mora, homes, culture.

Fecha de recepción: 17 de mayo, 2013 - Fecha de aceptación: 8 de agosto 2013

\begin{abstract}
Resumen
Este trabajo se basa en los datos de la encuesta realizada en dos comunidades del cantón de Puntarenas, uno de los principales centros históricos del turismo en el país, las cuales presentan grandes diferencias en sus orígenes y en la condición socioeconómica de sus pobladores, como son Juanito Mora, ubicada en el distrito de Barranca y El Barrio el Carmen, ubicada en el distrito central de Puntarenas y parte integral de lo que se conoce como el casco de la ciudad del mismo nombre. El objetivo de la investigación era determinar el impacto del turismo sobre estas comunidades y más específicamente se quería caracterizar a los hogares, conocer la composición de la población económicamente activa, conocer la fuente de los ingresos individuales y familiares y las opiniones que esa población tiene sobre el turismo, la cultura local y el papel de las instituciones locales en el desarrollo de esas comunidades. Esto con el objeto de proporcionar información a los tomadores de decisiones a nivel comunal. En este caso se va a comparar una serie de variables sociodemográficas y de opinión obtenidas en dos comunidades del mismo cantón, pero que presentan características diferentes, ubicadas en un foco de turismo nacional, para determinar cómo perciben los pobladores de dichas comunidades al turismo, como actividad económica y el aporte que la misma le brinda al desarrollo local.
\end{abstract}

\begin{abstract}
This work is based on data from the survey conducted in two communities from Puntarenas Central County, which is one of the main historical centers of tourism in the country. These communities present several differences from one another both social and economical, one is Juanito Mora, located in the district of Barranca and the other one is the central district of Puntarenas, which happens to
\end{abstract}


be an integral part of what is known as the old city holding the same name. The aim of the research was to determine the impact of tourism in these communities and more specifically, to characterize households, to know the composition of the economically active population, to identify the source of incomes and to know the opinions that people have regarding tourism, local culture and the role of local institutions in the development of these communities. This has the purpose of providing information to community decision makers. In order to determine how the residents of these regions perceive tourism and its benefits as an economical activity, a number of sociodemographic variables are to be compared with the opinion obtained from neighbors of these two communities: Juanito Mora and Puntarenas Central District. 


\section{ANÁLISIS COMPARATIVO DEL IMPACTO Y PERCEPCIONES SOBRE EL TURISMO DE LOS POBLADORES DE LAS COMUNIDADES DE BARRIO EL CARMEN Y JUANITO MORA EN PUNTARENAS, COSTA RICA. 2011}

Jorge Bartels Villanueva

\section{INTRODUCCIÓN}

Con este estudio se pretende hacer un aporte relevante en el conocimiento de las percepciones sobre el impacto y valoración de turismo, que tienen las comunidades localizadas en aquellas regiones donde esta actividad económica tiene un mayor desarrollo. Se quiere rescatar el enfoque regional desde una perspectiva comparativa, que nos permita trascender el análisis micro, "local" y monovariable, al posibilitar estudios multivariables, que a su vez proporcionen una imagen integradora y global de la dinámica que siguieron las diversas regiones del país en el desarrollo de la actividad turística, de forma que se pueda plantear una nueva interpretación del desarrollo de Costa Rica.

Este estudio complementa otro que se realizó en el 2011, en el cual se aplicó el mismo procedimiento a dos comunidades: Playas del Coco, ubicada en la provincia de Guanacaste y la comunidad del Roble 2 ubicada en el distrito del Roble en Puntarenas (Bartels, 2012, pp. 221-241). Los resultados de este primer experimento dejaron ciertas dudas, por lo que con el fin de esclarecerlas se decidió ampliar la investigación a dos comunidades más, ubicadas esta vez en el cantón de Puntarenas, Juanito Mora, localidad del distrito de Barranca, y Barrio el Carmen, situada en el casco central de Puntarenas.

Partimos del análisis micro, en este caso, las comunidades ya mencionadas, con la intención de ir agregando variables que permitieran una interpretación más integradora del aporte del turismo a las regiones y de la percepción que existe en estos lugares de esta actividad económica, para determinar si se generaliza ó si presenta variantes y cuáles son las posibles causas de esas interpretaciones o percepciones diversas.

Esta investigación tiene su origen en la idea de que Puntarenas, Limón y Guanacaste son los tres principales polos de desarrollo turístico del país. En primera instancia, Puntarenas y Limón se constituyen en los principales lugares de atracción de turistas, pero después, por diversas causas, empiezan a perder esa hegemonía y Guanacaste gana terreno a partir del desarrollo de muchos de sus poblados costeros, que disponen de bellas playas y que se ven favorecidos por la construcción y mejora de las vías de comunicación. 


\section{SE LECCIÓN DE LAS COMUNIDADES INVESTIGADAS}

Para el proceso de investigación se decidió comparar dos comunidades asociadas al polo de desarrollo turístico, de acuerdo con el modelo que se configuró desde su creación. En el caso de Puntarenas estamos hablando del modelo integrado $^{1}$, por oposición al modelo de enclave ${ }^{2}$. Lo anterior con el fin de comparar la información de dos comunidades con características diferentes y ubicadas, geográficamente, una en el centro del polo y otra en la periferia, para luego determinar el posible efecto de ambas sobre el modelo.

Uno de los objetivos específicos de este proyecto de investigación es medir el impacto del turismo en las comunidades ubicadas en lo que hemos denominado Centros Históricos del Turismo. En una primera fase los estudios se enfocaron, para el caso de Puntarenas, en la comunidad del Roble 2, ubicada en el distrito del mismo nombre y los resultados que se obtuvieron dejaron una gran duda, ya que no concordaban con las hipótesis planteadas (Bartels, 2012). El criterio experto que se consultó posteriormente indicaba que la población que vive en esta comunidad está directamente ligada a actividades del sector público y que se caracteriza por estar conformada, en su mayoría, por una población de clase media y condiciones socioeconómicas de nivel medio alto.

Ante tal situación se decidió contrastar esa información con dos comunidades ubicadas en Puntarenas y que representaran patrones diferentes a los ya analizados para el Roble.

A partir de este análisis se determinó que era necesario ubicar una comunidad que se encontrara inserta en el centro de la actividad económica de Puntarenas y una comunidad periférica, que presentara condiciones de marginalidad y cumpliera con la característica de ser proveedor de mano de obra para las actividades productivas de la zona.

La elección por Barrio El Carmen, como comunidad de aplicación del estudio de campo, estuvo asociada a su ubicación geográfica, pues se localiza directamente en el centro del polo turístico; también se consideró su tradición como destino fuente de actividades turísticas y las condiciones de transformación de un centro urbano de población a un centro de concentración de actividades comerciales y productivas. Esta comunidad ha estado ligada al turismo desde la creación misma del puerto de Puntarenas.

Producto del auge económico de los años 70 y 80 y de la conformación geográfica de la ciudad de Puntarenas, recordemos que es una isleta rodeada de mar por un lado y un gran estero por el otro, el crecimiento de la población se incrementó a tal grado que la ciudad debió extenderse hacia tierra firme y es así como aparecen comunidades como Barranca, Fray Casiano y Juanito Mora, donde se trasladan a vivir los hijos de los primeros habitantes de la ciudad y las personas que llegan atraídas por el crecimiento económico de la zona. 
El origen de esta comunidad está en la respuesta dada por el gobierno de Costa Rica a una serie de movimientos y presiones sociales que se dieron en la década de los ochentas, producto de las condiciones de marginalidad en que vivía un gran sector de la población de Puntarenas, aunado a los procesos migratorios del campo hacia la periferia de la ciudad y de los problemas de espacio que se enfrentaban en ésta, producto de su particular condición geográfica.

Esto factores motivaron al Estado a desarrollar proyectos habitacionales de bajo costo que permitieran dotar a las familias de una vivienda con ciertas condiciones mínimas y así resolver el problema social que enfrentaba. Lo anterior dio origen a la comunidad de Juanito Mora, nombrada así en memoria del expresidente de Costa Rica, que libró las batalla de 1856 y que es considerado un héroe nacional. La comunidad de Juanito Mora forma parte de una serie de barrios que se crearon bajo estas mismas condiciones y que presentan condiciones de marginalidad que aún no han podido ser solventadas.

Es así como la comunidad de Juanito Mora, ubicada en el distrito de Barranca de Puntarenas fue seleccionada porque reunía las condiciones de periferia del polo turístico, condiciones socioeconómicas de marginalidad y tener como principal fuente de actividad generadora de ingresos el trabajo de sus habitantes y no empresas ubicadas en la misma región. Es una comunidad básicamente residencial, compuesta por familias numerosas, provenientes en su mayoría del centro de Puntarenas, personas que se trasladaron a vivir a otras zonas, por la problemática de falta de espacio para nuevos hogares en la cabecera de la ciudad.

\section{DISEÑO Y SELECCIÓN MUESTRAL PARA EL PROYECTO³}

Este proyecto se sustentó en la información recopilada por medio de una encuesta elaborada por los miembros del equipo de investigación y que tenía por objetivo determinar el impacto y conocer las percepciones de los habitantes de las comunidades seleccionadas, sobre la actividad económica del turismo.

El diseño consistió en seleccionar dos comunidades de Puntarenas, en las cuales se reconoce que una parte de sus habitantes trabajan en actividades relacionadas con el turismo. Se eligieron las comunidades de Barrio El Carmen en Puntarenas Centro y el barrio Juanito Mora de Barranca. Con base en la distribución geográfica de ambos lugares, se procedió a preparar el marco de la muestra con visitas y recorridos, comparando con los mapas respectivos y actualizando la información en cada lugar.

Se determinó que el barrio Juanito Mora estaba conformado por 1097 viviendas y en el Barrio El Carmen se contabilizaron un total de 1422 viviendas. 


\section{a) Diseño muestral del barrio Juanito Mora}

Con base en los hogares o viviendas de esa comunidad, se decidió realizar un muestreo por conglomerados. De esta forma se definieron ocho conglomerados (cada conglomerado se conforma por una cantidad de viviendas ubicadas en manzanas o los llamados cuadrantes). Por tanto, el conglomerado, para nuestro estudio, corresponde a los llamados cuadrantes. El diseño muestral se realizó seleccionando cierta cantidad de viviendas en cada conglomerado.

Para la selección de las viviendas, primero se calculó el tamaño de la muestra que fue de 285 viviendas, equivalente a una fracción de muestreo de aproximadamente un $26 \%$. En cada conglomerado se escogieron 35 viviendas y se agregaron cinco más como posibles sustitutas de aquellas en las que por algún motivo no se pudiera aplicar la encuesta, ya sea porque no estuvieran habitadas o porque no se encontrara un informante adecuado para realizar la entrevista. Lo anterior nos da un total de cuarenta viviendas elegidas por conglomerado.

En cada conglomerado se seleccionaron aleatoriamente viviendas las cuales fueron visitadas y se entrevistó al jefe del hogar.

\section{b) Diseño de la muestra en barrio El Carmen}

El procedimiento seguido es similar al anterior. Se realizó un muestreo aleatorio de conglomerados y de cada uno se seleccionaron viviendas. El tamaño de la muestra, en este caso, fue calculado en 373 viviendas, es decir una fracción de muestreo de $26 \%$ aproximadamente.

Aquí se formaron 12 conglomerados y se escogieron aleatoriamente las viviendas en cada uno de ellos Se decidió seleccionar 31 viviendas en cada conglomerado y dejar cuatro viviendas más como posibles sustitutos para enfrentar los casos de ausencia de informante.

En cada conglomerado se visitaron las viviendas y se entrevistó al jefe de hogar.

La utilización de los conglomerados en ambos comunidades, estuvo determinada, entre otras razones por: las manzanas o cuadrantes por lo general son muy heterogéneos, las viviendas están frecuentemente juntas, por lo tanto la cercanía era un factor facilitador.

Era posible obtener mayor información muestreando un número grande de conglomerados de menor tamaño, sin embargo se tomaron viviendas de todos los conglomerados.

A continuación se detalla el procedimiento de la selección de viviendas en cada conglomerado, por medio de un muestreo aleatorio sistemático:

\section{c) Cálculo del tamaño de la muestra}

\section{1- $\alpha=0.95 \Rightarrow Z=1.96$ (95\% de confianza para las estimaciones obtenidas)}


$\mathrm{d}=$ Margen de error $=4 \%$ (4\% de error es las estimaciones obtenidas)

$\mathrm{P}=$ Proporción de personas que se dedican al turismo o se benefician de esa actividad.

$\mathrm{P}=0.70$ ( $70 \%$ con base en una estimación realizada)

$$
\begin{array}{ll}
n=\left[\frac{1.96 \sqrt{0.70(0.30)}}{0.04}\right]^{2} \quad & \Rightarrow
\end{array}
$$

Una fracción de muestreo de $26 \%$

Se tomaron todos los conglomerados y se seleccionaron viviendas de cada uno en forma sistemática; se seleccionó el número de viviendas en cada conglomerado, pero se dejó una cantidad viviendas como sustitutas, para los casos en que el hogar visitado no estuviera habitado o no se encontrara alguien para suministrar la información.

\section{ANÁLISIS DE RESULTADOS}

\section{Tabla 1}

COMPOSICIÓN DE LA MUESTRA

\begin{tabular}{lccc}
\hline \multirow{2}{*}{ Localidad } & \multicolumn{2}{c}{ Número de personas } & \% de personas en el \\
\cline { 2 - 3 } & Entrevistadas & En el sector turismo & \\
\hline Puntarenas Centro & 309 & 39 & $12.62 \%$ \\
Juanito Mora & 209 & 13 & $6.22 \%$ \\
\hline
\end{tabular}

Fuente: Elaboración propia.

Específicamente se trabajó con una muestra de 309 hogares de Barrio El Carmen de Puntarenas y de 209 hogares del barrio Juanito Mora, para un total 
de 518 hogares de ambas comunidades y una población total de 799 individuos pertenecientes a éstos.

\section{Tabla 2}

CARACTERÍSTICAS LABORALES DE LOS TRABAJADORES, SEGÚN UBICACIÓN DEL LOCAL (2011)

\begin{tabular}{lcc}
\hline Variable & Puntarenas Centro & Juanito Mora \\
\hline Horas de trabajo a la semana (Cantidad) & 53.0263 & 45.8182 \\
Antigüedad laboral (en años) & 7.16 & 5.77 \\
Porcentaje que cotiza al seguro social & $84.2 \%$ & $41.7 \%$ \\
Porcentaje que recibe capacitación & $47.4 \%$ & $53.8 \%$ \\
\hline
\end{tabular}

Fuente: Elaboración propia.

Al realizar las pruebas de comparación de medias para los dos grupos se concluye con un $95 \%$ de confianza que:

- La cantidad de horas trabajadas a la semana promedio es mayor para los trabajadores de Barrio El Carmen que para los de Juanito Mora.

- $\quad$ La antigüedad laboral promedio es mayor en 1.39 años para los trabajadores de Barrio El Carmen que para los de Juanito Mora.

- $\quad$ El porcentaje de trabajadores que cotizan para el Seguro Social es significativamente mayor en Barrio El Carmen que en Juanito Mora.

- $\quad$ El porcentaje de trabajadores que recibe capacitación es ligeramente inferior en Barrio el Carmen que en Juanito Mora.

Aunque las variables muestran un comportamiento ligeramente positivo para el Barrio El Carmen, excepto en lo que se refiere a la capacitación recibida por los trabajadores, llama la atención que en él existe un número mucho mayor de trabajadores cotizando para el Seguro Social, lo cual los pone en ventaja frente a los trabajadores de Juanito Mora. 


\begin{tabular}{lcc}
\hline \multicolumn{1}{c}{ Variable } & \multicolumn{2}{c}{ Turismo } \\
\cline { 2 - 3 } & Puntarenas Centro & Juanito Mora \\
\hline $\begin{array}{l}\text { Número de personas que trabajan en el } \\
\text { establecimiento (Promedio) }\end{array}$ & 24.19 & 4.60 \\
$\begin{array}{l}\text { Porcentaje de establecimientos con dueño } \\
\text { procedente del cantón }\end{array}$ & $57.1 \%$ & $55.6 \%$ \\
$\begin{array}{l}\text { Porcentaje de establecimientos con dueño nacional } \\
\text { pero no del cantón }\end{array}$ & $22.9 \%$ & $22.2 \%$ \\
& & \\
Porcentaje de establecimientos dueño extranjero & $20.0 \%$ & $22.2 \%$ \\
\hline
\end{tabular}

Fuente: Elaboración propia.

Al realizar las pruebas de comparación de medias para los dos grupos se concluye con un $95 \%$ de confianza que:

- $\quad$ El número de personas promedio que trabajan en el establecimiento es mucho mayor para la localidad de Barrio El Carmen que para Juanito Mora.

- $\quad$ No existe suficiente evidencia estadística para aceptar la hipótesis de que el porcentaje de establecimientos con dueño procedente del cantón es diferente para las dos localidades, ya que el porcentaje es casi el mismo en ambas.

- Existe suficiente evidencia estadística para aceptar la hipótesis de que el porcentaje de establecimientos con dueño nacional pero no del cantón es igual para las dos localidades.

- $\quad$ El porcentaje de establecimientos con dueño extranjero es igual para ambas localidades.

Lo anterior, ya que no se encontraron diferencias significativas entre las localidades para las variables dos tres y cuatro. Lo que sí se puede destacar es que las personas de Barrio El Carmen que laboran en el sector turismo lo hacen en establecimientos de mayor tamaño, que contratan mayor cantidad de personal. 


\begin{tabular}{lccc}
\hline & & \multicolumn{2}{c}{ Turismo } \\
\cline { 3 - 4 } & Variable & Puntarenas Centro & Juanito Mora \\
\cline { 3 - 4 } & Hombre (\%) & 64.1 & 46.2 \\
Sexo & & & 53.8 \\
& Mujer (\%) & 35.9 & 34.15 \\
Edad (años) & & 42.84 & 8.15 \\
Escolaridad (años) & & 9.76 & \\
\hline
\end{tabular}

Fuente: Elaboración propia.

Al realizar las pruebas de comparación de medias para los dos grupos se concluye con un $95 \%$ de confianza que:

- $\quad$ El porcentaje de hombres y mujeres que laboran en el sector turismo es mayor en Barrio El Carmen que en Juanito Mora.

- $\quad$ La edad promedio de los trabajadores de Juanito Mora es menor que la de los trabajadores de Barrio El Carmen.

- Existe suficiente evidencia estadística para rechazar la hipótesis que la escolaridad es igual para las dos localidades, pues se demuestra que la escolaridad es mayor para los trabajadores de Barrio El Carmen.

Es interesante ver que el porcentaje de hombres que labora en el sector turismo, provenientes de Barrio El Carmen es mayor que el porcentaje de mujeres de esa misma comunidad y que el correspondiente a los hombres de Juanito Mora. En la comunidad de Juanito Mora se da la situación inversa, la cantidad de mujeres que laboran en el sector turismo es mayor que la cantidad de hombres.

También se puede observar que la población laboral del sector turismo que vive en Juanito Mora es más joven que la de Barrio El Carmen, lo cual se podría explicar por el origen de estas comunidades. 


\section{Tabla 5}

CARACTERÍSTICAS SOCIODEMOGRÁFICAS DE LOS JEFES DE HOGAR DE LAS FAMILIAS

DE TRABAJADORES, SEGÚN UBICACIÓN DEL LOCAL (2011)

\begin{tabular}{|c|c|c|c|}
\hline \multicolumn{2}{|l|}{ Variable } & \multirow{2}{*}{$\begin{array}{c}\text { Puntarenas Centro } \\
58.6\end{array}$} & \multirow{2}{*}{$\begin{array}{c}\text { Juanito Mora } \\
33.3\end{array}$} \\
\hline & Hombre $(\%)$ & & \\
\hline \multicolumn{4}{|l|}{ Sexo del jefe } \\
\hline & Mujer (\%) & 41.4 & 66.7 \\
\hline Edad del jefe (años) & & 45.1379 & 34.8000 \\
\hline Escolaridad del jefe (en años) & & 8.4483 & 6.4000 \\
\hline & Ocupado (\%) & 93.1 & 50.0 \\
\hline \multicolumn{4}{|l|}{ Condición de actividad del jefe } \\
\hline & No ocupado $(\%)$ & 6.9 & 50.0 \\
\hline
\end{tabular}

Fuente: Elaboración propia.

Al realizar las pruebas de comparación de medias para los dos grupos se concluye con un $95 \%$ de confianza que:

- $\quad$ El porcentaje de hombres y mujeres jefes de hogar es igual para ambas localidades, lo único que cambia es que en Juanito Mora vamos a encontrar un mayor número de mujeres jefas de hogar.

- $\quad$ Existe suficiente evidencia estadística para rechazar la hipótesis de que la edad es igual para las dos localidades, se demuestra que la edad promedio del jefe de hogar es mayor para Barrio El Carmen.

- Existe suficiente evidencia estadística para rechazar la hipótesis de que la escolaridad de los jefes de hogares es igual para las dos localidades, se demuestra que este índice es mayor para los trabajadores jefes de hogar de Barrio El Carmen.

- $\quad$ Existe suficiente evidencia estadística para rechazar la hipótesis de que el porcentaje de los jefes de hogares ocupados es igual para las dos localidades, se encuentra que el porcentaje es mayor para los jefes de hogar de Barrio El Carmen. 
Es notorio que los jefes de hogar que laboran en el sector turismo provenientes de Barrio El Carmen se encuentran en una situación ventajosa en lo que se refiere a la escolaridad y la condición de su actividad laboral, es decir presentan una mayor escolaridad y un mayor número de ellos se encuentra empleado, lo cual es preocupante para la comunidad de Juanito Mora, ya que se demostró que existe una mayor cantidad de mujeres jefas de hogar y una mayor desocupación.

\section{Tabla 6}

CARACTERÍSTICAS LOS DE HOGARES DE LAS FAMILIAS DE TRABAJADORES, SEGÚN UBICACIÓN DEL LOCAL (2011)

\begin{tabular}{lcc}
\hline \multicolumn{1}{c}{ Variables } & Puntarenas Centro & Juanito Mora \\
\hline Tamaño del hogar(número promedio de personas) & 3.0345 & 2.9000 \\
Relación de dependencia demográfica & .3155 & .1667 \\
Relación de dependencia laboral & .4802 & .4481 \\
Total de ingresos mensuales $(\phi)$ & 416.880 & 240.000 \\
Ingresos mensuales per capital $(\phi)$ & & 98.472 \\
\hline
\end{tabular}

Fuente: Elaboración propia.

Al realizar las pruebas de comparación de medias para los dos grupos se concluye con un $95 \%$ de confianza que:

- El tamaño del hogar es mayor en la comunidad de Barrio El Carmen.

- Existe suficiente evidencia estadística para rechazar la hipótesis de que la relación de dependencia laboral es igual para las dos localidades, se demuestra que es mayor para los hogares de Juanito Mora.

- $\quad$ Existe suficiente evidencia estadística para rechazar la hipótesis de que el total de ingresos mensuales es igual para las dos localidades, se encuentra que es mayor para los hogares de Barrio El Carmen. 
- $\quad$ Existe suficiente evidencia estadística para rechazar la hipótesis de que el ingreso mensual per cápita es igual para las dos localidades, se encuentra que es mayor para los hogares de Juanito Mora.

- La relación de dependencia demográfica es mayor para el Barrio El Carmen.

Es interesante ver cómo a pesar que los datos de Juanito Mora tienden a ser menos favorables que los del Barrio El Carmen, en aspectos como el tamaño del hogar y las relaciones de dependencia laboral y demográfica; el primero logra una pequeña ventaja, por lo menos para los trabajadores ubicados en el sector turismo. En cuanto a los ingresos promedios y per cápita, los pobladores de Juanito Mora siguen en situación de desventaja.

Tabla 7

OPINIÓN DE LOS ENTREVISTADOS SOBRE EL TURISMO Y SU IMPACTO,

SEGÚN UBICACIÓN DEL LOCAL (2011)

\begin{tabular}{|c|c|c|c|}
\hline \multicolumn{2}{|c|}{ Variables } & \multirow[t]{2}{*}{ Puntarenas Centro } & \multirow[t]{2}{*}{ Juanito Mora } \\
\hline \multirow{3}{*}{$\begin{array}{l}\text { El turismo ha impactado en el } \\
\text { cantón }(\%)\end{array}$} & Nada y poco & & \\
\hline & Más o menos & 70.1 & 89,1 \\
\hline & Mucho y totalmente & 29.9 & 10.9 \\
\hline \multirow{3}{*}{ Valoración del impacto (\%): } & Malo y muy malo & 20.50 & 22.7 \\
\hline & Neutral & 34.7 & 46.4 \\
\hline & Bueno y muy bueno & 44.8 & 30.9 \\
\hline
\end{tabular}

Fuente: Elaboración propia.

Al realizar las pruebas de comparación de medias para los dos grupos se concluye con un $95 \%$ de confianza que: 
Existe suficiente evidencia estadística para rechazar la hipótesis de que el porcentaje de hogares que opinan que el turismo no ha impactado nada es igual para las dos localidades, se encuentra que es mayor para los hogares de Juanito Mora.

- $\quad$ Existe suficiente evidencia estadística para rechazar la hipótesis de que el porcentaje de hogares que opinan que el turismo ha impactado más o menos es igual para las dos localidades, se encuentra que es mayor para los hogares de Juanito Mora.

- $\quad$ Existe suficiente evidencia estadística para rechazar la hipótesis de que el porcentaje de hogares que opinan que el turismo ha impactado mucho es igual para las dos localidades, se encuentra que es mayor para Barrio El Carmen.

- $\quad$ El porcentaje de hogares que piensa que el turismo ha impactado de forma negativa es igual para ambas localidades

- $\quad$ Existe suficiente evidencia estadística para rechazar la hipótesis de que el porcentaje de hogares que opina que el turismo ha impactado de forma neutral es igual para las dos localidades, se encuentra que es mayor para los hogares de Juanito Mora.

- $\quad$ Existe suficiente evidencia estadística para rechazar la hipótesis de que el porcentaje de hogares que opina que el turismo ha impactado de forma positiva es igual para las dos localidades, se encuentra que es mayor para los hogares de Barrio El Carmen.

Los resultados del cuadro muestran que en la comunidad de Juanito Mora, los entrevistados, en un mayor porcentaje, consideran que el impacto del turismo ha sido neutral, es decir que no ha provocado efectos positivos ni negativos. Las opiniones negativas sobre el impacto del turismo, aunque no son mayoritarias, también están más marcadas en la comunidad Juanito Mora.

Por otra parte, los pobladores de los hogares de Barrio El Carmen presentan una valoración más positiva en lo relativo al impacto del turismo en su comunidad y le otorgan una evaluación más positiva a los efectos del turismo sobre el cantón.

\section{CONCLUSIONES}

Sobre el impacto del turismo, aunque no hay diferencias significativas entre los datos de las dos comunidades, se puede decir, en general, que hay una posición positiva con respecto al turismo, a pesar de que no son muchas las personas que están en esta actividad.

Estas dos comunidades, aunque tienen un origen distinto: la primera es el lugar tradicional de visitación de turistas, sobre todo nacionales; y la segunda surge como producto de soluciones a problemas habitacionales de la zona y como respuesta a 
las demandas de vivienda de los sectores más marginales de Puntarenas, ven en el turismo una actividad económica importante para la generación de empleo y si bien consideran que puede representar aspectos negativos, no le cierran la puerta del todo.

Encontramos que en la comunidad de Juanito Mora, la cantidad de hogares relacionados con esta actividad es más pequeña que en el Barrio El Carmen, pero los habitantes en ambas comunidades parecen presentar más puntos en común que diferencias. Se debe mencionar que los aspectos en los cuales se detectan mayores diferencias en las características de los hogares de los encuestados, se refieren a:

- $\quad$ Escolaridad.

- Edad de los jefes de hogar.

- La condición de actividad de los jefes de hogar.

- $\quad$ El ingreso promedio y el ingreso per cápita recibido.

Sobre la valoración y el impacto del turismo, se encuentran diferencias entre las dos comunidades.

En la comunidad de Juanito Mora se tiene una percepción de que el turismo como actividad económica ha impactado poco al cantón, y no hay una clara definición sobre si el impacto ha sido positivo o negativo.

En Barrio el Carmen hay una valoración relativamente más favorable del impacto de la actividad turística en el cantón, pues tiende a ser más positiva, lo cual puede atribuirse, como ya se mencionó anteriormente, a que esta comunidad se ubica en el corazón de la actividad turística de Puntarenas.

A la hora de medir los efectos del turismo, los informantes de ambas comunidades manifestaron que se debe incrementar esta actividad, ya que es buena para el desarrollo económico local. La mayoría opina que el desarrollo del turismo es importante para el cantón, a pesar de que también indicaron que el turismo desplaza a los nacionales, pues estos venden sus propiedades a los extranjeros, quienes adquieren privilegios en cuanto al acceso a los servicios públicos y que además promueve la prostitución.

Es decir, parece que el turismo se valora positivamente en cuanto que genera recursos al interior de los hogares, pero tiene una connotación negativa por los efectos que produce en la sociedad donde se desarrolla, es decir, causa externalidades positivas para los individuos y negativas para la sociedad. Es interesante que las opiniones obtenidas en este estudio coincidan, en gran medida, con las que se registraron al aplicar el mismo cuestionario a los pobladores de las comunidades de Playas del Coco en Guanacaste y del Roble 2 en Puntarenas. Esto nos hace concluir que en todos estos lugares se presentan más puntos de coincidencia que discrepancias.

Por otra parte, este estudio, al realizar un ajuste en las categorías laborales estudiadas, permitió estimar porcentajes de población de estas comunidades muy 
cercanos a las cifras que manejan los entes oficiales, como el Instituto Costarricense del Turismo, que presenta cifras cercanas al $7 \%$ de empleo para el sector. Consideramos que no existe una clara conciencia de los impactos del turismo y que estos aún no han sido cuantificados en sus verdaderos alcances.

Como se mencionó en ocasiones anteriores, quedan pendientes otros estudios sobre este asunto, y en esta dirección se han enfocado las actividades que desarrollan muchas unidades académicas e investigadores que trabajan el tema del turismo en la Universidad de Costa Rica y fuera de ella.

La información recolectada en esta investigación y los materiales elaborados (cuadros y bases de datos) están a disposición de investigadores, estudiantes e instituciones, entre otros. Esta información, al ser tan variada y cubrir dos diferentes regiones del país, abre una gran gama de posibilidades de investigación sobre temas diversos y con enfoques metodológicos novedosos, que sin lugar a dudas enriquecerán la compresión de nuestro desarrollo.

\section{CITAS Y NOTAS}

1 El modelo Integrado propicia el desarrollo de pequeños y medianos emprendimientos, se conserva la base territorial autóctona, el turismo de base comunitaria es más extendido, la identidad local se conserva relativamente o se da una hibridez cultural y las modificaciones ambientales se dan de una forma más integrada.

2 El modelo de Enclave se caracteriza porque el desarrollo local se basa casi exclusivamente en la generación de empleos, el impacto socio-territorial ocasiona la perdida de una base territorial autóctona, existe un escaso desarrollo de la base comunitaria, reducido al encadenamiento de grandes empresas, se produce una pérdida de la identidad local y una entronización de las culturas turísticas globalizadas, y las modificaciones sobre la base natural son profundas.

3 En este diseño colaboró el Lic. Orlando Vásquez Rodríguez, quien también participó en el proceso de levantamiento de la información.

\section{BIBLIOGRAFÍA}

Bartels, J. (2012). Aportes del turismo según los habitantes de las comunidades de Playas del Coco, Guanacaste y el Roble Puntarenas. Diálogos, Volumen especial, 227-242.

Chen, S. (2012). Elementos históricos del desarrollo del turismo en Puntarenas. Diálogos, Volumen especial, 121-150.

Chen, S. y García, K. (2007). Puntarenas y el turismo: ¿Qué ha pasado con la «Perla del Pacífico»? Inter Sedes, VIII(15), 109-131. 
Chen, S. y García, K. (2010). Percepción del impacto del turismo en el Roble 2 de Puntarenas, Costa Rica. Reflexiones, 89(2), 27-38.

Cordero, A. (2008). Los viejos y olvidados centros históricos del turismo: algunas evidencias para el caso costarricense. XLV colloque de l'Asociation descience régionale de la langue Francaise. [XLV conferencia de la Asociación de ciencia regional de la lengua francesa]. CR: FLACSO.

Cordero, A. (2013). Allá en playas del Coco; donde el turismo no fue amor de temporada. Inter Sedes, XI(22), 154-179.

Gatgens, G. (2010). Puntarenas. La Perla del Pacífico. http://www.puntarenas-cr.com/histsimb.htm

\section{ACERCA EL AUTOR}

Jorge Bartels Villanueva: Magister en Economía, Profesor e investigador, Sede del Pacífico de la Universidad de Costa Rica 
